\title{
Interference Aware Throughput Measurements for Mobile WiMAX over Vehicular Radio Channels
}

\author{
Bjoern Dusza, Christoph Ide and Christian Wietfeld \\ Communication Networks Institute \\ TU Dortmund University \\ 44227 Dortmund, Germany \\ e-mail: \{Bjoern.Dusza, Christoph.Ide, Christian.Wietfeld\}@tu-dortmund.de
}

\begin{abstract}
Due to its advantageous properties in multipath propagation scenarios, the Orthogonal Frequency Division Multiple Access (OFDMA) radio transmission scheme is widely spread in current broadband wireless networks. Exemplary emerging systems based on this technology are the UMTS Long Term Evolution (LTE), Mobile WiMAX and some popular WiFi variants (e.g. IEEE 802.11n). Although analytical and simulative investigations have shown that the increased symbol time of OFDMA (compared with single carrier systems) allows for a wider delay spread without causing Inter Symbol Interference (ISI) it is quite hard to quantify this improvement under realistic circumstances.

In this paper, we present the results of a measurement campaign in which we investigated the influence of multipath fading channels, interferences and velocity on the available UDP data rate of an IEEE 802.16e conform Mobile WiMAX System. Therefore, a sophisticated laboratory setup was used which is based on a radio channel emulator. From that, optimum switching points for the Adaptive Modulation and Coding (AMC) scheme are derived. The results show, that for vehicular radio channels the choice of the most suitable modulation and coding scheme, and therefore the maximum available data rate, does strongly depend on the environmental parameter such as delay spread and user velocity. In particular, it can be seen that the use of a strong forward error correction is more important than a higher order modulation scheme if high data rates are required by real time multimedia applications.
\end{abstract}

\section{INTRODUCTION}

Although the LTE system seems to win the race on becoming the next widely spread wireless communication technology, there are some very interesting applications for the competitive Mobile WiMAX Technology. One major advantage of Mobile WiMAX is the quite compact core network which allows for smaller sized systems for special applications. Two recently investigated scenarios for which Mobile WiMAX can be applied are the distribution of IPTV content [1] as well as the connection of fast trains to the Internet [2]. One major advantage of the OFDMA based Mobile WiMAX system is its robustness against impairments as they occur on the mobile radio channel. This includes fading due to multipath propagation as well as high delay spreads, interferences and Doppler-Shifts due to high speed mobility. Although the impact of these effects on the radio link performance has been extensively investigated by means of simulations and analytical investigations it is quite difficult to reliably measure key performance indicators such as the

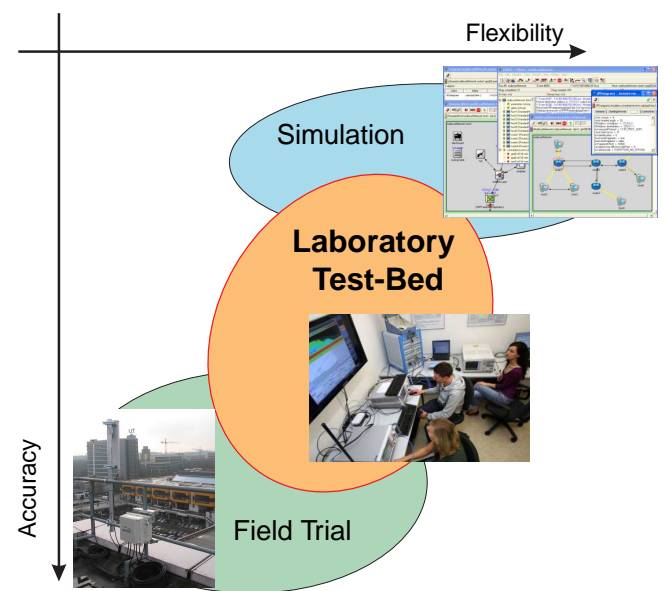

Fig. 1. Approaches for the Assessment of Wireless Communication Systems

data rate using real hardware under reproducible channel conditions. Fig. 1 illustrates the benefits and drawbacks of different approaches for the performance evaluation of mobile communication systems in realistic environments. While simulations allow for a high degree of flexibility, the necessary assumptions and simplified models may lead to a lack of accuracy. On the other hand field trails allow for a maximum degree of accuracy but most of the system parameters are either fixed (such as the base station parameterization) or uncontrollable (e.g. the radio channel and the cell utilization). In this paper, we present the results of a measurement campaign that is based on laboratory measurements which may close the gap between the previously described approaches. A highly sophisticated radio channel emulator was used to introduce effects such as fast fading, interferences and Doppler shifts to the radio link between a Base Station Emulator (BSE) and an User Equipment (UE). Based on this setup, measurements of the data rate for different ITU channel models, different signal to interference and noise ratios (SINR) and different user velocities have been performed for various modulation and coding schemes (MCS) assuming an IEEE 802.16e Mobile WiMAX conform radio link. From that, scenario dependent optimum switching points between the different MCS were derived. 


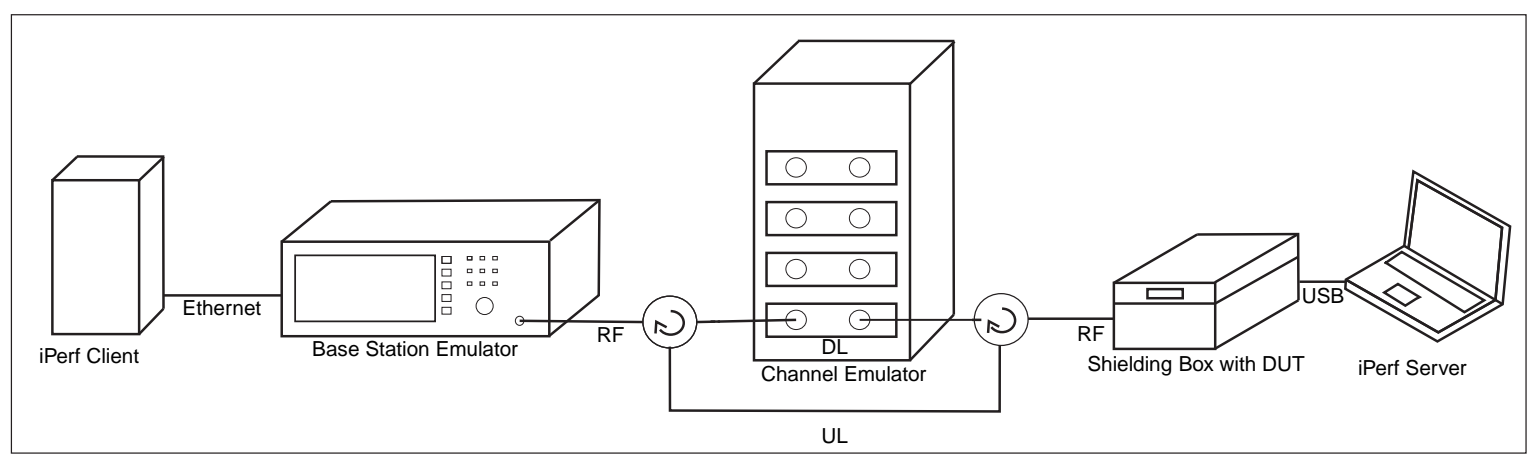

Fig. 2. Measurement Setup for Bidirectional Performance Testing

\section{RELATED WORK}

An interesting approach for a performance evaluation of WiMAX links with respect to throughput measurements can be found in [3], where the channel is realized by using a channel emulator with Stanford University Interim (SUI) channel models and an additional air interface. Although the approach is promising, in this paper it is very hard to distinguish which effects are due to the emulator and which ones occur on the additional radio link.

Beside the channel emulation approach, the evaluation of OFDMA based links is typically performed by means of simulation or field trials (see Fig. 1). In [4] the performance of Mobile WiMAX is analyzed using a physical layer simulation. Here, the authors focus on the characteristics of the throughput and the PER for different channel models and various modulation and coding schemes. These investigations are particularly made for the ITU vehicular A channel model assuming different velocities. Beside this, in [5] a technology comparison between LTE and WiMAX with focus on throughput simulations for different user velocities (ITU vehicular A model) can be found.

On the other hand real-world measurements are frequently performed for the analysis of the impact of velocity on the performance of OFDMA based links. For example the performance of LTE is evaluated by a test bed in [6]. Here, for throughput measurements a monitoring car with an average speed of about $30 \mathrm{~km} / \mathrm{h}$ was used. Nevertheless, this method has the drawback that it is quite difficult to drive with a constant and preset speed to precisely measure the influence of the velocity. Hence, in field trials static scenarios [7] or scenarios with low velocity are usually considered ([8]; pedestrian $3 \mathrm{~km} / \mathrm{h}$ fading channels in downlink and static channels in uplink).

In [9] investigations with a fully compliant Mobile WiMAX simulator are compared with experimental results from field measurements. Therefore, the throughput and the Packet Error Rate (PER) for different modulation and coding schemes are evaluated for 3GPP channel models. It is worth noting that in most cases it is assumed that the optimum choice of the MCS does only depend on the SINR that it measured at the receiver. Tab. I shows the assumed receiver SINR at the MCS switching points from the mobile WiMAX standard [10]. One can see that the influences of the building density as well as the user velocity on the optimum MCS switching point is not considered by the current version of the standard.

\section{Measurement Setup}

The overall measurement setup used for the evaluation of the channel dependent User Datagram Protocol (UDP) downlink throughput of Mobile WiMAX can be seen in Fig. 2. The iPerf client at the left hand side of the figure creates the UDP traffic that is transferred to a standard conform Mobile WiMAX Base Station Emulator via Ethernet. This device acts as a gateway and transfers the data to a Mobile WiMAX RF signal at $3.5 \mathrm{GHz}$. Via an RF cable this signal is feed to the radio channel emulator where it is manipulated according to the preset channel conditions. Fig. 3 illustrates the most important modifications of the signal inside the channel emulator. More details on the channel emulator can be found in [11].

In a first step, the undisturbed input signal is transferred to the baseband and converted to the digital domain. Here, the signal is convoluted with the time variant impulse response corresponding to the previously defined channel model. The multipath characteristic of the channel leads to the frequency selective nature of the channel transfer function which can be seen in Fig. 3.2. Beside this, the signal is spread according to the velocity dependent Doppler spread.

After these modifications of the signal the channel emulator allows for the addition of adjustable Additive White Gaussian Noise (AWGN) interference at the output of the device (Fig. 3.3). Therefore, the signal power is measured and noise according to a predefined SINR is created and added to the

TABLE I

RECEIVER SINR ASSUMPTIONS FROM IEEE 802.16E STANDARD [10]

\begin{tabular}{|c|c|c|}
\hline Modulation & Coding Rate & Receiver SINR \\
\hline \multirow{2}{*}{ QPSK } & $1 / 2$ & 3.0 \\
& $3 / 4$ & 8.5 \\
\hline \multirow{2}{*}{ 16-QAM } & $1 / 2$ & 11.5 \\
& $3 / 4$ & 15.0 \\
\hline \multirow{2}{*}{ 64-QAM } & $1 / 2$ & 19.0 \\
& $3 / 4$ & 21.0 \\
\hline
\end{tabular}




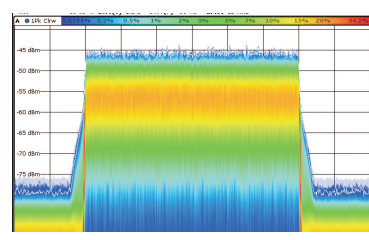

1. Undisturbed input OFDMA Signal from Base Station

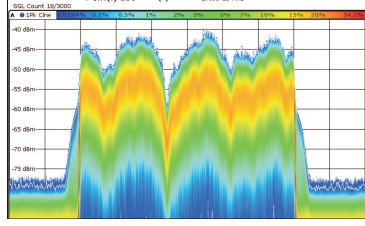

2. Frequency Selective Fading (here ITU Veh. A)
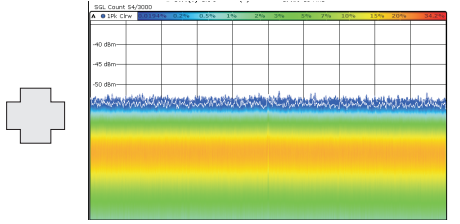

3. Adjustable AWGN

Corresponding to Given SINR

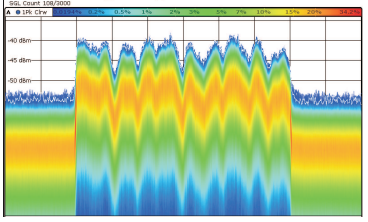

4. Receiving Signal of the UE (With Fading and Interference)

Fig. 3. Manipulation of an OFDMA Input Signal by the Radio Channel Emulator (captured by real time spectrum analyzer)

signal. This dynamic process allows for a constant SINR even if the signal power changes over time. In this case, the noise power will be automatically adjusted for meeting the SINR requirement.

At the output of the channel emulator one can find a faded and interfered version of the input signal which is spread according to the velocity dependent Doppler shift (Fig. 3.4). This signal is transferred to the user equipment with the iPerf server via RF cable. Due to the fact that for the measurements presented at this paper only the downlink is of relevance, for the uplink the channel emulator is bypassed by circulators and another RF cable (see Fig. 2).

\section{Measurement CAmpaign}

For the emulation of the mobile radio channel the ITU vehicular A and pedestrian B channel models according to [12] are used for the downlink channel.

For the considered ITU radio channels "Classical" fading models are used. They make use of the Rayleigh amplitude distribution and Jakes-Doppler spectrum. The Rayleigh probability density function $p_{R a}$ of amplitude $r$ is therefore given by [13]

$$
p_{R a}(r)=\frac{r}{\sigma^{2}} \exp \left(-\frac{r^{2}}{2 \sigma^{2}}\right)
$$

where $\sigma^{2}$ denotes the variance of both the real and imaginary components of the signal independently. In the classical model all incident angles are assumed to occur equally distributed, leading to the normalized Doppler power spectrum formula defined below [13].

$$
S(f)=\frac{1}{\pi f_{d} \sqrt{1-\left(\frac{f}{f_{d}}\right)^{2}}}
$$

In this formula $f_{d}$ denotes the maximum Doppler frequency shift depending on the carrier frequency $f_{c}$, the speed of light $c$ and the velocity of the user $v$.

$$
f_{d}=f_{c} \cdot \frac{v}{c}
$$

From the Doppler shift the influence of mobility is introduced to the channel transfer function and therefore impacts the transmitted Mobile WiMAX signal by spreading the impulse power in the frequency domain. For the measurement campaign presented in this paper the ITU channel model, the user velocity and the SINR are modified at the radio channel emulator. Beside this, the modulation and coding scheme
(MCS) is adjusted at the base station emulator. The overall parametrization of the Mobile WiMAX base station emulator is given in Table II.

For the evaluation of the data rate an iPerf UDP throughput test was performed for each modulation and coding scheme, an emulated SINR ranging from $0 \mathrm{~dB}$ to $30 \mathrm{~dB}$ and the two different channel models described earlier. Beside this, an additional measurement was performed for which only the emulated velocity of the user equipment was modified for an ITU vehicular A channel while a fixed SINR of $30 \mathrm{~dB}$ was used.

\section{RESUlts}

In this section, the results of the different measurements are presented in detail. In all of the plots the continuous lines represent a scheme with code rate of $1 / 2$ while the dashed line shows an MCS with code rate of 3/4. The bold line in each of the plots shows the modulation and coding scheme for which the best performance can be achieved for the various SINR and velocities. Fig. 4 illustrates the data rate as a function of the SINR for different modulation and coding schemes assuming an ITU pedestrian B radio channel [12]. As it can be seen from the plot, only four out of the six considered modulation and coding schemes should be used for achieving the maximum data rate. For very low SINR of up to $8 d B$ the QPSK $1 / 2$ is the best choice and allows for a data rate of about $2.2 \mathrm{Mbit} / \mathrm{s}$. From $8 d B$ to $17 d B$ SINR the highest data rate of up to $7 \mathrm{Mbit} / \mathrm{s}$ is achievable by means of the 16-QAM 1/2. The 64-QAM 1/2 offers the best results for SINR from $17 d B$ to $24 d B$ while only for very good interference conditions

TABLE II

MobILE WiMAX SYSTEM PARAMETRIZATION

\begin{tabular}{|c|c|}
\hline Parameter & Value \\
\hline Carrier Frequency $[\mathrm{GHz}]$ & 3.5 \\
\hline Channel Bandwidth $[\mathrm{MHz}]$ & 10 \\
\hline Transmitter Power $[\mathrm{dBm}]$ & -15 \\
\hline Sampling Frequency $1 / T_{s}$ & $11.2 \mathrm{MHz}$ \\
\hline FFT Size $N_{F F T}$ & 1024 \\
\hline Modulation Schemes & QPSK, 16-QAM, 64-QAM \\
\hline Coding Rates & $1 / 2,3 / 4$ \\
\hline Cyclic Prefix (CP) Length $G$ & $1 / 8$ \\
\hline Coding Type & Convolutional Turbo Code (CTC) \\
\hline Duplexing Scheme & TDD (DL/UL Ratio: 35:12) \\
\hline Carrier Interleaving Scheme & FUSC \\
\hline MIMO Type & 1 x 1 (SISO) \\
\hline Map Repetition Factors & 0 (No Repetition) \\
\hline SINR & 0 - 30 dB \\
\hline
\end{tabular}




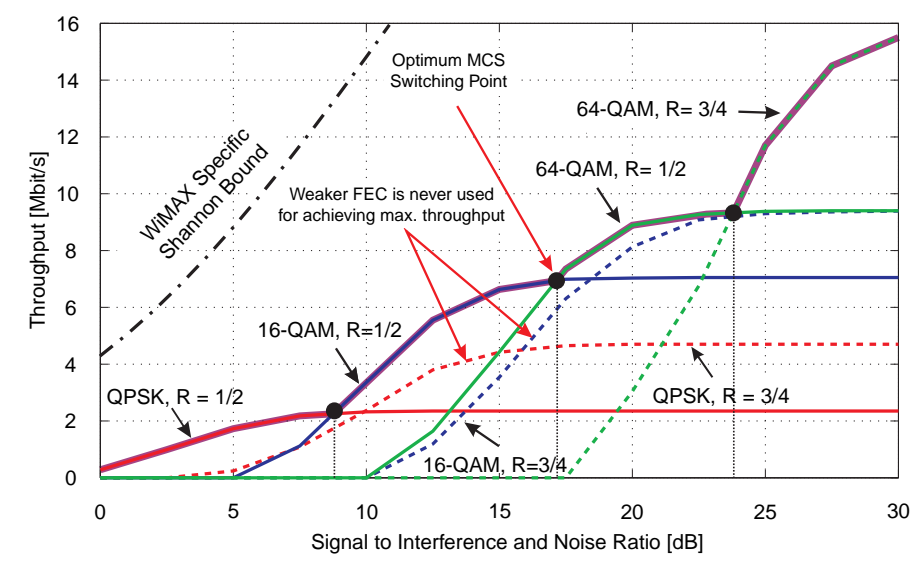

Fig. 4. Throughput for different SINR (ITU Ped. B Channel @ $3 \mathrm{~km} / \mathrm{h}$ )

with more than $24 d B$ SINR the 64-QAM 3/4 allows for the maximum data rate which is about $15.5 \mathrm{MBit} / \mathrm{s}$ for an SINR of $30 d B$. It should be noted that the use of the QPSK 3/4 as well as the 16-QAM 3/4 does not make any sense from a maximum data rate perspective.

In addition to the measurement results, a theoretically derived maximum data rate $D_{\max }$ under pure AWGN conditions (WiMAX Specific Shannon Bound) is shown. This can be calculated based on [14]

$$
D_{\max }=\frac{1}{1+G} \cdot \frac{1 / T_{s}}{N_{F F T}} \cdot \frac{N_{D L}-3}{N_{\text {all }}} \cdot I
$$

with the extension that three symbols of the DL portion $N_{D L}$ are used by control information [15] and therefore have to be subtracted. The parameter $G$ denotes the length of the $\mathrm{CP}, 1 / T_{s}$ is the signal sample frequency and $N_{F F T}$ refers to the FFT Size. The value $N_{\text {all }}$ represents the total number of OFDMA symbols in one subframe. The concrete values used for Fig. 4 are given in Tab. II. The mutual information $I$ of an AWGN channel is given as

$$
I=\log _{2}(1+S N R)
$$

where SNR denoted the signal to noise ratio.

The ITU vehicular channel models represent channel conditions as they occur in typical vehicular environments

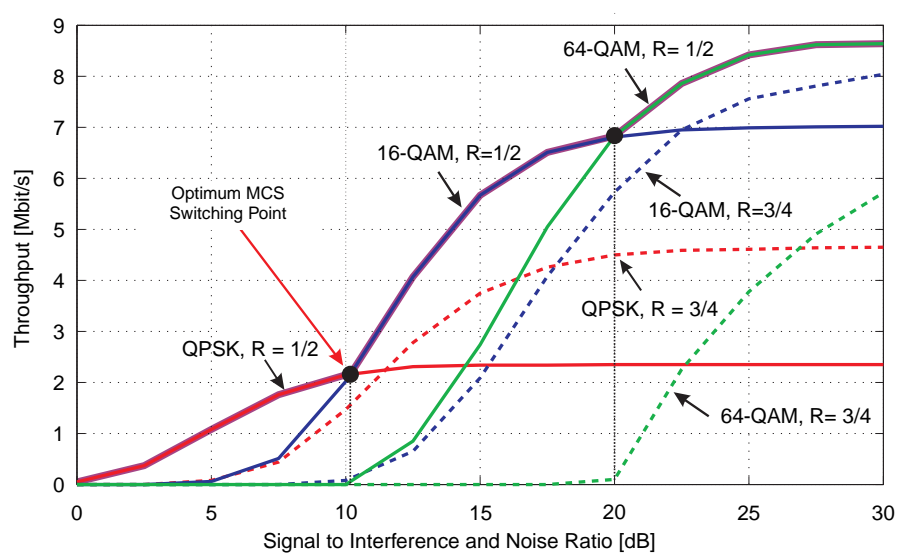

Fig. 5. Throughput for different SINR (ITU Veh. A Channel @ 60 km/h)

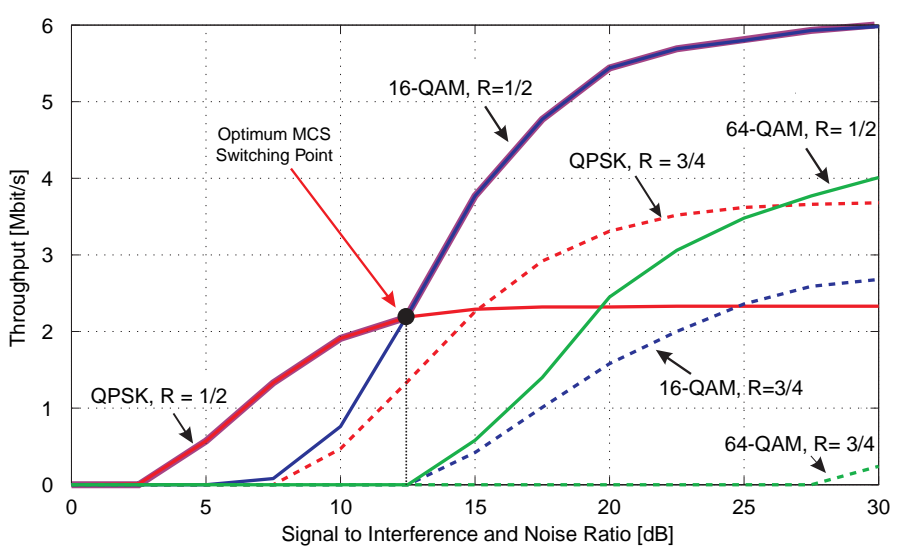

Fig. 6. Throughput for different SINR (ITU Veh. A Channel @ 120 km/h)

assuming higher velocities. Fig. 5 shows the results of the measurement for a vehicular A channel assuming a user velocity of $60 \mathrm{~km} / \mathrm{h}$. As it can be seen from the plot, the modulation and coding schemes with a code rate of $3 / 4$ are of no relevance for achieving a maximum data rate. For very low SINR of up to $10 d B$ the QPSK $1 / 2$ is the best choice. For better interference conditions with SINR of up to $20 d B$ the 16-QAM 1/2 outperforms all other schemes and for very high SINR of more than $20 d B$ the 64-QAM $1 / 2$ is the most suitable modulation and coding scheme. It has to be noted, that also for very high SINR of up to $30 d B$ the maximum achievable data rate is only 8.6 Mbit/s for an ITU vehicular A channel. The impact of velocity on the data rate can be seen from Fig. 6 where the emulated velocity is increased to $120 \mathrm{~km} / \mathrm{h}$ for a vehicular A channel. For this scenario only two modulation and coding schemes are used for achieving the maximum possible data rate. For SINR of up to $12.5 d B$ the QPSK 1/2 allows for the best performance while for higher SINR the 16-QAM $1 / 2$ is the most suitable choice. All other schemes incl. the 64-QAM 1/2 should not be used if the maximum possible throughput is required. The maximum achievable data rate over a vehicular A channel at $120 \mathrm{~km} / \mathrm{h}$ under optimal interference conditions (30 dB SINR) is $6 \mathrm{Mbit} / \mathrm{s}$. The plot in Fig. 7 shows the pure impact of velocity on the data rate assuming the ITU vehicular A channel model. For this measurement the AWGN interference was set to

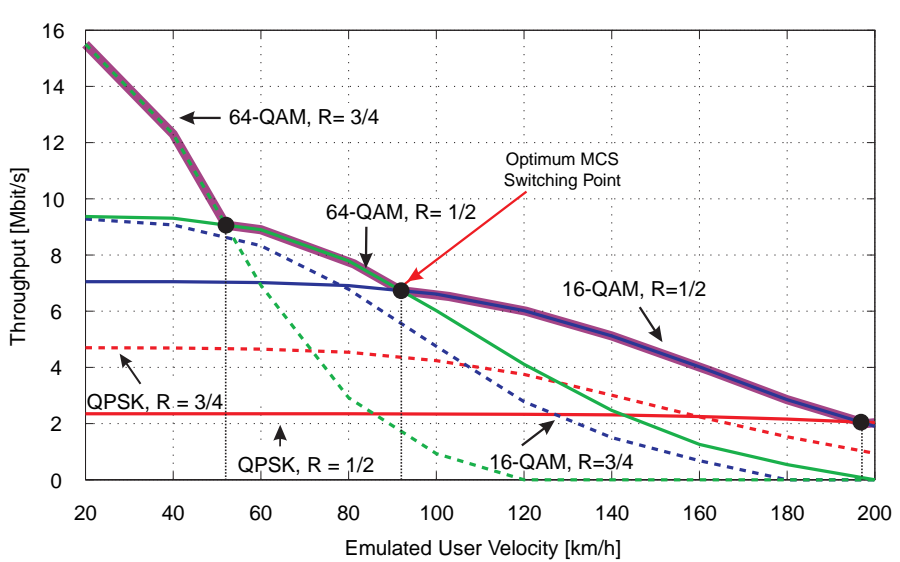

Fig. 7. Throughput for different velocities @ $30 \mathrm{~dB}$ SINR (ITU Veh. A cha.) 


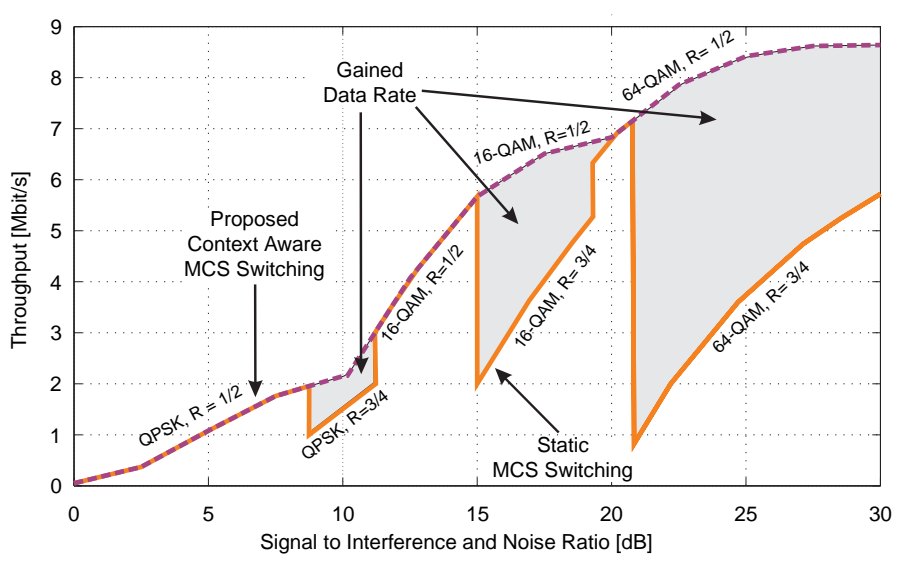

Fig. 8. Comparison of Channel Aware AMC with Static Approach from Standard [10] (exemplary for ITU Vehicular A Channel @ $60 \mathrm{~km} / \mathrm{h}$ ))

$30 d B$ and the user speed was varied from $20 \mathrm{~km} / \mathrm{h}$ to $200 \mathrm{~km} / \mathrm{h}$. As it can be seen from the plot, for low velocities the maximum throughput can be achieved by means of a 64-QAM 3/4. Nevertheless, the performance of this scheme decreases very fast with increasing user speed. Assuming a user that accelerates his car to a speed of up to $200 \mathrm{~km} / \mathrm{h}$ while he is maintaining a UDP connection the best choice of the modulation and coding scheme would be as follows: Up to a speed of $55 \mathrm{~km} / \mathrm{h}$ the 64-QAM 3/4 allows for the highest throughput. For velocities of $55 \mathrm{~km} / \mathrm{h}$ to $90 \mathrm{~km} / \mathrm{h}$ the 64-QAM $1 / 2$ is the best choice and for higher velocities the mobile user should switch to a 16-QAM 1/2. For velocities of more than $200 \mathrm{~km} / \mathrm{h}$ the QPSK $1 / 2$ is the most suitable MCS.

\section{Context Aware vs. Static SinR Thresholds FOR ADAPTIVE MODULATION AND CODING}

From all of the previously described result plots one can see, that the optimum switching points between the different MCS are strongly depending on the radio channel characteristics. Therefore, we propose to choose the AMC switching points context aware based on implicitly available meta information such as environment and user velocity instead of applying fixed SINR thresholds as proposed in the standard [10] (see Tab. I). Fig. 8 illustrates the performance improvements that can be achieved by the context aware approach for an ITU vehicular A channel at a user velocity of $60 \mathrm{~km} / \mathrm{h}$. One can see from the comparison of the different schemes that the static approach usually switches to a higher order MCS at a too low SINR. This leads to a significantly decreased data rate for some SINR areas. The impact of this effects becomes worse the more fading is observed on the channel.

\section{CONCLUSION AND FURTHER WORK}

In this paper, we have presented the results of extensive measurements on the impact of fading channels, AWGN interferences and user velocity on the maximum achievable data rate of a Mobile WiMAX system. The results show that for vehicular channels and especially at high velocities the choice of a robust Forward Error Correction (FEC) code with a code rate of $1 / 2$ is more important than a low order modulation scheme if high data rates have to be achieved.

Beside this, we have shown that the suitable choice of a MCS does strongly depend on the channel characteristics as well as the user velocity while the relationship can not be analytically modeled based only on the SINR. Therefore the optimum switching points should not be statically chosen based on predefined SINR values (see Tab. I) but context aware based on meta information such as user velocity, environment and QoS requirements. Our next step will be the investigation of concrete cross layer approaches for achieving this context awareness.

\section{ACKNOWLEDGMENT}

Part of the work on this paper has been supported by Deutsche Forschungsgemeinschaft (DFG) within the Collaborative Research Center SFB 876 "Providing Information by Resource-Constrained Analysis", projects A4 and B4.

\section{REFERENCES}

[1] J. M. Lee, H.-J. Park, S. G. Choi, J. K. Choi, Adaptive Hybrid Transmission Mechanism for On-Demand Mobile IPTV Over WiMAX, IEEE Transactions on Broadcasting, VOL. 55, No. 2, June 2009

[2] K. R. Kumar, P. Angolkar, D. Das, R. Ramalingam, SWiFT: A Novel Architecture for Seamless Wireless Internet for Fast Trains, Proc. of the IEEE 67th Vehicular Technology Conference (VTC), Marina Bay, Singapore, May 2008

[3] Schwengler, T. Pendharkar N., Testing of fixed broadband wireless systems at $5.8 \mathrm{GHz}$, Proc. of the Technical, Professional and Student Development Workshop, 2005 IEEE Region 5 and IEEE Denver Section, Denver, Apr. 2005

[4] R. Colda, T. Palade, E. Puschita, I. Vermesan, A. Moldovan, Mobile WiMAX: System Performance on a Vehicular Multipath Channel, Proc. of the Fourth European Conference on Antennas and Propagation (EuCAP), Barcelona, Apr. 2010

[5] C. Ball, T. Hindelang, I. Kambourov, S. Eder, Spectral Efficiency Assessment and Radio Performance Comparison Between LTE and WiMAX, Proc. of the IEEE 19th International Symposium on Personal, Indoor and Mobile Radio Communications (PIMRC), Cannes, Sep. 2008

[6] N. Miyazaki, S. Nanba, S. Konishi, MIMO-OFDM Throughput Performances on MIMO Antenna Configurations Using LTE-Based Testbed with $100 \mathrm{MHz}$ Bandwidth, Proc. of the IEEE 72st Vehicular Technology Conference (VTC), Ottawa, Sep. 2010

[7] H. Oguma et al., Uplink Throughput Performance of FH-OFMDA Improved by 16 QAM: Effect Estimation and Validation in MBWA System Field Trial, Proc. of the IEEE 20th International Symposium on Personal, Indoor and Mobile Radio Communications (PIMRC), Tokyo, Sep. 2009

[8] R. Irmer et al., Multisite Field Trial for LTE and Advanced Concepts, IEEE Communications Magazine, Feb. 2009

[9] M. Tran, G. Zaggoulos, A. Nix, A. Doufexi, Mobile WiMAX: Performance Analysis and Comparison with Experimental Results, Proc. of the IEEE 68th Vehicular Technology Conference (VTC), Calgary, 2008

[10] Part 16:Air Interface for Broadband Wireless Access Systems, IEEE Std 802.16-2009, May 2009

[11] C. Ide, B. Dusza, C. Wietfeld, Mobile WiMAX Performance Measurements with Focus on Different QoS Targets, in Proc. of the 18th IEEE International Workshop on Local and Metropolitan Area Networks (LANMAN), Chapel Hill, USA, 2011

[12] International Telecommunication Union, Recommendation ITU-R M.1225 Guidelines for Evaluation of Radio Transmission Technologies for IMT-2000, 1997

[13] M. Paetzold, Mobile Fading Channels, Wiley, England, 2002

[14] C. Mehlfuehrer, S. Caban, M. Rupp, Experimental Evaluation of Adaptive Modulation and Coding in MIMO WiMAX with Limited Feedback, EURASIP Journal on Advances in Signal Processing, Vol. 2008, Article ID 837102, 2008

[15] D. Pareit, V. Petrov, B. Lannoo, E. Tanghe, W. Joseph, I. Moerman, P. Demeester, L. Martens, A Throughput Analysis at the MAC Layer of Mobile WiMAX, Proc. of the Wireless Communications and Networking Conference (WCNC), Sydney, Apr. 2010 